genden neuronalen Netzwerke und chemischen Signalwege notwendig, um eine gezielte und wirkungsvolle Behandlung zu ermöglichen.

Chronischer Juckreiz kann dermatologische, systemische, psychogene oder neuropathologische Ursachen haben. Schädigungen des Nervensystems, die zu einem neuropathischen Juckreiz oder zu neuropathischen Schmerzen führen, können durch verschiedene Erkrankungen wie multiple Sklerose, Herpes zoster oder Diabetes mellitus, aber auch durch Schlaganfälle hervorgerufen werden. Viele Patienten leiden an Geschwüren und Infektionen durch das ständige Kratzen.

\title{
Klinische Präsentation
} und Diagnose

Kann für einen chronischen Juckreiz keine dermatologische Ursache gefunden und eine systemische Ursache anhand der Krankengeschichte ausgeschlossen werden, sollte der Patient auf einen neuropathischen Juckreiz hin untersucht werden. Dieser kann in verschiedenen Ausprägungen auftreten, die sich in Ursachen und Symptomen deutlich unterscheiden:

Großflächige periphere Small-Fiber-Neuropathien verursachen einen großflächigen Juckreiz an den Extremitäten (proximal zu distal steigend). Die Ursachen reichen von der Behandlung mit Hydroxyethylstärke über Mutationen im NaV1.7-Natriumkanal bis zum Ehlers-Danlos-Syndrom.

\section{Neuropathischer Juckreiz: klinische Präsentation, Diagnose und Therapie}

Steinhoff M et al. Clinical presentation, management, and pathophysiology of neuropathic itch. Lancet Neurol 2018; 17: 709-720

Neuropathischem Juckreiz liegt eine Schädigung peripherer oder zentraler Nerven zugrunde. Die verschiedenen Ursachen und Formen des neuropathischen Juckreizes machen eine eindeutige Identifikation schwierig. Es mangelt an standardisierten Definitionen und Untersuchungsmethoden. Zudem ist die Erforschung der zugrunde lie-
Fokale periphere Mononeuropathien, Plexopathien und Radikulopathien treten vorwiegend am Kopf und oberen Torso sowie an den Armen auf. Häufigste Ursache ist eine Schädigung der Nervenwurzel - in Hautbiopsien kann dann eine unzureichende Innervierung nachgewiesen werden. Weitere mögliche Ursachen sind Herpes zoster, mikrovaskuläre Komplikationen infolge einer Diabetes-mellitus-Erkrankung oder eine multiple endokrine Neoplasie Typ 2.

Spinale sensorische Gangliopathien erzeugen einen Juckreiz, der nicht entlang der Nervenbahnen verläuft und häufig in Verbindung mit neuropathischen Schmer- 
zen und Ataxie auftritt. Die Symptome können auf eine Krebserkrankung, Infektion oder Autoimmunerkrankung hindeuten und bedürfen sofortiger Behandlung.

Läsionen im Gesicht, Kopf und Nacken können durch Verletzungen des Ganglion trigeminale verursacht werden. Die häufigste Ursache für neuropathischen Juckreiz im Gesicht ist Herpes zoster.

Läsionen des zentralen Nervensystems, beispielsweise durch einen Schlaganfall, können ebenfalls zu neuropathischem Juckreiz, häufig gepaart mit neuropathischem Schmerz und Gefühlsverlust, führen.

Tritt der Juckreiz in Verbindung mit einem Gefühlsverlust auf, deutet dies auf eine neurologische Ursache hin. Die Krankengeschichte des Patienten kann Aufschluss über mögliche Ursachen einer Neuropathie wie Diabetes mellitus oder Herpes zoster geben. Mithilfe sensorischer Tests (wie beispielsweise den von Frey Haaren) kann der Juckreiz eingegrenzt und lokal behandelt werden. Neurologische Ursachen und Nervenverletzungen können mit PGP9.5-Antikörpern bzw. anhand einer Elektromyografie aufgespürt werden. Standardisierte Fragebögen zum chronischen Juckreiz helfen bei der Diagnose und der Dokumentation des Krankheitsverlaufs.

\section{Therapie}

Die Behandlung neuropathischen Juckreizes zielt auf die Vermeidung und Behandlung von Kratzwunden ab. Eine psychotherapeutische Betreuung kann zusätzlich dabei helfen, das Kratzverlangen zu unterdrücken und Depressionen und Aggressivität entgegenzuwirken.

Peripherer Juckreiz wird zumeist lokal behandelt - Lidocain-Salbe und subkutane Injektionen von Anästhetikum können den Juckreiz unterbinden, Capsaicin wird zur Degeneration der Nervenenden verwendet. Bei großflächigem neuropathologischem Juckreiz hilft eine systemische Medikation mit Natriumkanalblockern. Häufig kommen auch Opioide und Antidepressiva zum Einsatz.

\section{FAZIT}

Die Behandlung von neuropathischem Juckreiz stellt eine große Herausforderung dar. Der Mangel an eindeutigen Bewertungskriterien erschwert die Diagnose und da die Krankheitsmechanismen nicht völlig verstanden sind, fehlen effiziente Therapieansätze. In Tiermodellen konnten verschiedene zelluläre Netzwerke identifiziert werden, die bei neuropathischem Juckreiz eine Rolle spielen und deren weitere Erforschung neue Therapieansätze hervorbringen kann.

Dr. Astrid Weiler, Emsdetten 